\title{
Stage Related Mortality in Carcinoma Oesophagus Treated by Surgery
}

\author{
Harisankar Ananthapurath Girijavallabann ${ }^{1}$, Muhammed Basheer Ottathayil ${ }^{2}$ \\ ${ }^{1}$ Senior Resident, Department of General Surgery, Government Medical College, Kozhikode, Kerala, India. \\ ${ }^{2}$ Associate Professor, Department of General Surgery, Government Medical College, Kozhikode, Kerala, India.
}

\section{ABSTRACT}

\section{BACKGROUND}

Oesophageal carcinoma is the eighth most common cancer, and the sixth most common cause of cancer related deaths worldwide with developing nations making up more than $80 \%$ of total cases and deaths. Despite many advances in diagnosis and treatment, the 5-year survival rate for all patients diagnosed with oesophageal cancer ranges from $15 \%$ to $20 \%$. The objective of the study was to know the survival rate of patients in relation to stage at presentation in carcinoma oesophagus.

\section{METHODS}

This is a retrospective cohort study conducted over a period of 5 years in 50 patients who underwent oesophagectomy for carcinoma oesophagus in the Department of Surgery, Govt. Medical College, Kozhikode. The patients were followed up based on details obtained from Medical Records Library of Government Medical College, Calicut, and the present status was obtained after reviewing them on OP basis. Chi square test was used for statistical analysis. The study was approved by Institutional Ethics Committee.

\section{RESULTS}

Mean age of patients who presented with carcinoma oesophagus is 59.3 years, with male to female ratio of 1.89:1. Majority of the patients had history of smoking. $98 \%$ presented with dysphagia. Major pre-operative risk factors are hypoalbuminemia followed by CAD and COPD. The mean survival days for stage $1,2, \& 3$, were 1147 days (37.7 months), 859 (28.24 months) days and 667 days (21.9 months) respectively. Mean survival days of patients with oesophageal malignancy in our study is 799.68. The difference between mean survival days between stage 1 and 3 and that between stage 2 and 3 were statistically significant with p value of 0.021 and 0.010 respectively, thus emphasizes the necessity of early detection and diagnosis. The mean hospital stay of TTE was more than THE. Other factors which determined the survival rate are hypoalbuminemia, immediate post-operative complications, and chemotherapy.

\section{CONCLUSIONS}

Considerable controversy continues regarding the best surgery for a given patient, though the procedure that the surgeon can perform safely is certainly the best. Since stage at presentation is a significant factor determining the survival of patients with oesophageal malignancy, early detection and diagnosis can significantly improve survival rate.

\section{KEY WORDS}

Oesophageal Malignancy, Survival Rate, Mean Survival Days
Corresponding Author:

Harisankar Ananthapurath Girijavallaban, Siva Ponnayil House,

Ananthapurath Lane,

Thammanam P. O. Kochi-682032,

Kerala, India.

E-mail: harisankar099@gmail.com

DOI: $10.14260 /$ jemds/2019/599

Financial or Other Competing Interests: None.

How to Cite This Article:

Harisankar AG, Basheer OTM. Stage related mortality in carcinoma oesophagus treated by surgery. J. Evolution Med. Dent. Sci. 2019;8(35):2763-2767, DOI: $10.14260 /$ jemds/2019/599

Submission 06-07-2019, Peer Review 18-08-2019, Acceptance 24-08-2019, Published 02-09-2019. 


\section{BACKGROUND}

Oesophageal carcinoma is the eighth most common cancer, and the sixth most common cause of cancer related deaths worldwide with developing nations making up more than $80 \%$ of total cases and deaths. ${ }^{1}$ The lifetime risk of oesophageal cancer in the United States is about 1 in 125 in men and about 1 in 454 in women. ${ }^{2}$ While many other types of cancer are expected to decrease in incidence over the next 10 years, by 2025 the prevalence of oesophageal cancer is expected to increase by $140 \% .^{3}$

Oesophageal cancer is considered a serious malignancy with respect to prognosis and mortality rate accounting for more than 400000 deaths worldwide in 2005.2 Despite many advances in diagnosis and treatment, the 5 -year survival rate for all patients diagnosed with oesophageal cancer ranges from $15 \%$ to $20 \% .4$

The epidemiology of oesophageal cancer in developed nations has dramatically changed. Squamous cell carcinoma (SCC) was responsible for greater than $90 \%$ of the cases of oesophageal carcinoma in the United States over the past forty years. Adenocarcinoma has now become the leading cause of oesophageal cancer in the United States, representing $80 \%$ of cases. ${ }^{5}$ Considerable differences of incidence of oesophageal cancer exist on the basis of geographic and racial differences, which can be linked to differences in exposure to risk factors. ${ }^{6}$

Surgical resection is the mainstay of therapy. Recent treatment strategies have included multimodality approaches that combine surgery, radiation therapy, and chemotherapy. These approaches have resulted in 5-year survival rates of $40 \%$ to $75 \%$ in the subset of patients who have a complete histologic response after preoperative therapy. ${ }^{7}$

The purpose of this study is to know the survival rate of patients in relation to stage at presentation and thus emphasizes the necessity of early detection and diagnosis.

\section{METHODS}

This was a retrospective cohort study over a period of 5 years involving 50 patients who underwent oesophagectomy for Carcinoma oesophagus, in the Department of Surgery, Govt. Medical College, Calicut between time period of 2011-2013. Patients were followed up based on details obtained from Medical Records Library of Government Medical College Calicut. The present status of the patients was obtained after reviewing them on OP basis. This study was approved by the ethics committee of Government Medical College, Calicut.

\section{Inclusion Criteria}

Patients with carcinoma oesophagus who could tolerate physiological resection, we also chose to include in the definition all tumour located at the $\mathrm{Z}$ line or within an area of $5 \mathrm{~cm}$ orally and $5 \mathrm{~cm}$ aborally of the anatomic junction between the oesophageal and gastric walls. Patients initially deemed unresectable, who had good response to neoadjuvant chemotherapy and subsequently underwent surgery were included.

\section{Exclusion Criteria}

Patients with evidence of distant metastasis and proximal one third lesions were not considered. Type 3 Siewerts were classified as Gastric cancers. The details of preoperative evaluation like chest Roentgenography, barium swallow, endoscopy with biopsy, bronchoscopy, in some cases computed tomography of the chest and operative and postoperative details were obtained

\section{Statistical Test}

Chi square test.

\section{RESULTS}

Most of the study subjects fall in the age group $>60$ years. The youngest being $36 \mathrm{yrs}$. and the oldest $72 \mathrm{yrs}$. of age. Mean age of our study is 59.3 years. Study group comprised of 36 (72\%) males and $14(28 \%)$ females, making M:F ratio of 1.89:1. This ratio compared favourably to the data for India based on the International agency for research on cancer (1.98:1).

Smoking is a prominent risk factor in oesophageal malignancies. Of the sample population $56 \%$ had history of cigarette smoking. $13 \%$ had history of alcoholism. As majority of the patients were well past their middle age, many of them had co-morbidities like HTN, DM and COPD. Of the sample population, $30 \%$ hypertensive, $28 \%$ were diabetic, $12 \%$ had history of pulmonary tuberculosis, $22 \%$ had history of COPD, $26 \%$ had CAD history.

Majority of the patients presented with dysphagia and the mean duration of onset of dysphagia to the surgery was 4.6 months comparable to 5-6 months reported by studies in Asia-Pacific region. High levels of literacy and good access to public health care in Kerala which is on par with most developed nations may be the reason.

$48 \%$ of cases $(24 / 50)$ had malignancy in mid third and $52 \%(26 / 50)$ in lower third of oesophagus. Frequency of histopathological type of oesophageal malignancy shows $40 \%$ of adenocarcinoma (20 patients) and $60 \%$ of squamous cell carcinoma (30 patients).

Carcinoma lower oesophagus had a mean survival of 889.65 days (2.43 yrs) which was higher compared to carcinoma mid oesophagus which is 702.2 days (1.92 yrs), but not statistically significant(p value -0.232 ). Majority of mid oesophageal malignancy presented in Stage 3 and most of the lower oesophageal malignancies were in Stage 2.

Majority of cases, $52 \%$, presented in stage III.

\begin{tabular}{|c|c|c|}
\hline Stage & Frequency & Percent \\
\hline 1 & 7 & 14.0 \\
\hline 2 & 17 & 34.0 \\
\hline 3 & 26 & 52.0 \\
\hline Total & $\mathbf{5 0}$ & $\mathbf{1 0 0 . 0}$ \\
\hline \multicolumn{2}{|c|}{ Table 1. Table Showing Frequency of Stage at Presentation }
\end{tabular}

In our study the mean survival days of patients with oesophageal malignancy in our study is 799.68 . For stage 1,2 \& 3 it was 1147 days (37.7 months), 859 (28.24 months) days and 667 days (21.9 months) respectively. 


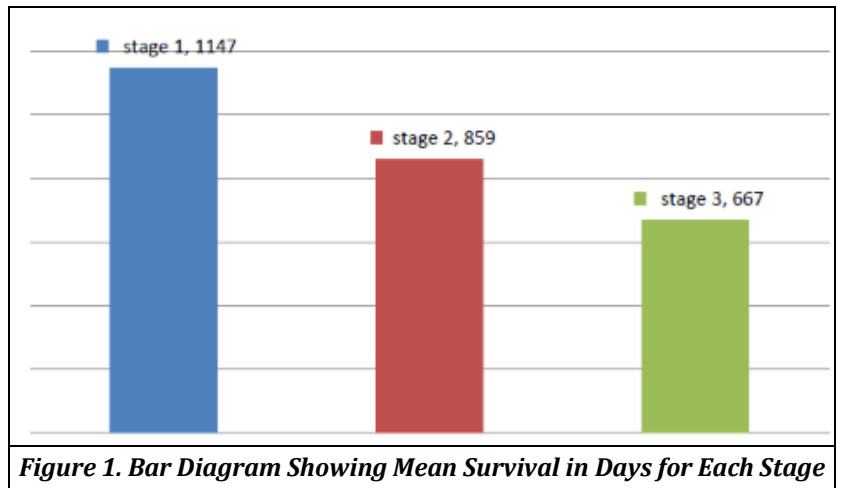

Mean survival days of patients with oesophageal malignancy in our study is 799.68. oesophageal malignancy diagnosed and treated at Stage 1 has survival advantage of 1.6 years compared to Stage 3 . This showed a p value of 0.021 and was statistically significant. Stage 2 oesophageal malignancy has survival difference of 1.3 yrs more than stage 3 and was statistically significant, $p$ value 0.010 . However, Stage 1 and 2 has only difference in survival of 116 days which is comparable.

The histological types of malignancy in $60 \%$ cases (30) was squamous cell carcinoma and 40\% (20) adenocarcinoma. Majority of lesions in mid oesophagus were squamous and presented in stage 3. In lower oesophagus $42.5 \%$ were in stage 2, most were adenocarcinoma. It was statistically significant with a p value of 0.001 . In our study, the mean survival days were 773 days for adenocarcinoma and 817 days for squamous cell carcinoma, which were comparable and not statistically significant.

$30 \%$ of the study population underwent tri-incisional oesophagectomy which included both thoracotomy and thoracoscopic approaches. $20 \%$ of the study population underwent Transhiatal oesophagectomy (THE). In our study the mean survival rate in patients after transthoracic approach was 2.34 yrs. compared to Transhiatal which was 1.85 years.

About $28 \%$ of patients had postoperative hospital stay for $\leq 13$ days and $56 \%$ had hospital stay for $>13$ days. Longer hospital stay was noted in all cases of transthoracic procedure.

\section{Post-Surgical Complications}

\begin{tabular}{|c|c|c|}
\hline Complications & Frequency & Percent \\
\hline Respiratory complications & 9 & 18 \\
\hline Anastomotic leak & 5 & 10 \\
\hline Pulmonary embolism & 4 & 8 \\
\hline Cardiac complication & 9 & 18 \\
\hline \multicolumn{2}{|c|}{ Table 2. Frequency of Post-Operative Complications } \\
\hline
\end{tabular}

The mean survival of patients with complications was significantly reduced (424.33 days) compared to those without complications (960.54), statistically significant ( $p$ value 0.001). Those patients with any immediate complications had a reduced survival compared to those with no immediate postop complications

Complications were present in $26.7 \%$ of cases who underwent Tri-incisional procedure and $35 \%$ cases who underwent THE.

$10 \%$ of patients ( 5 in no.) had serum albumin level $<3.5$ and $90 \%$ (45) had albumin levels $\geq 3.5$. All the patients with preoperative serum albumin level $<3.5$ had anastomotic leak, was statistically significant with $\mathrm{p}$ value 0.001 .

\section{Chemotherapy}

Out of 50 patients 15 underwent chemotherapy. The average survival of patients treated with chemotherapy and surgery was 34 months, compared to 22 months for patients treated with surgery alone

\begin{tabular}{|c|c|c|c|c|c|}
\hline Chemotherapy & N & Mean & SD & $\begin{array}{c}\text { Mean } \\
\text { Difference }\end{array}$ & p Value \\
\hline No & 35 & 693.97 & 558.468 & 352.362 & 0.036 \\
\hline Yes & 151046.33 & 454.770 & \\
\hline
\end{tabular}

Out of 15 patients who received chemotherapy $60 \%$ of them were Stage 3 and $40 \%$ Stage 2.The mean survival of Stage 3 with chemotherapy and surgery was 811 days compared to 419.35 with surgery alone.

Following surgery, out of the 50 patients 9 patients could not be followed up due to immediate death. $57 \%$ of the patients had Dysphagia score of 3, 15\% had fairly good dysphagia score of 1 .

Comparing preoperative and postoperative weight of patients, there was mean post-operative weight gain of $1.3 \mathrm{~kg}$ on long term follow up.

Survival analysis was done with Kaplan Meir method. The median survival time in patients undergoing oesophagectomy is 1095 days (36 months) with a $95 \%$ confidence interval 696.3 to 1493.7 days.

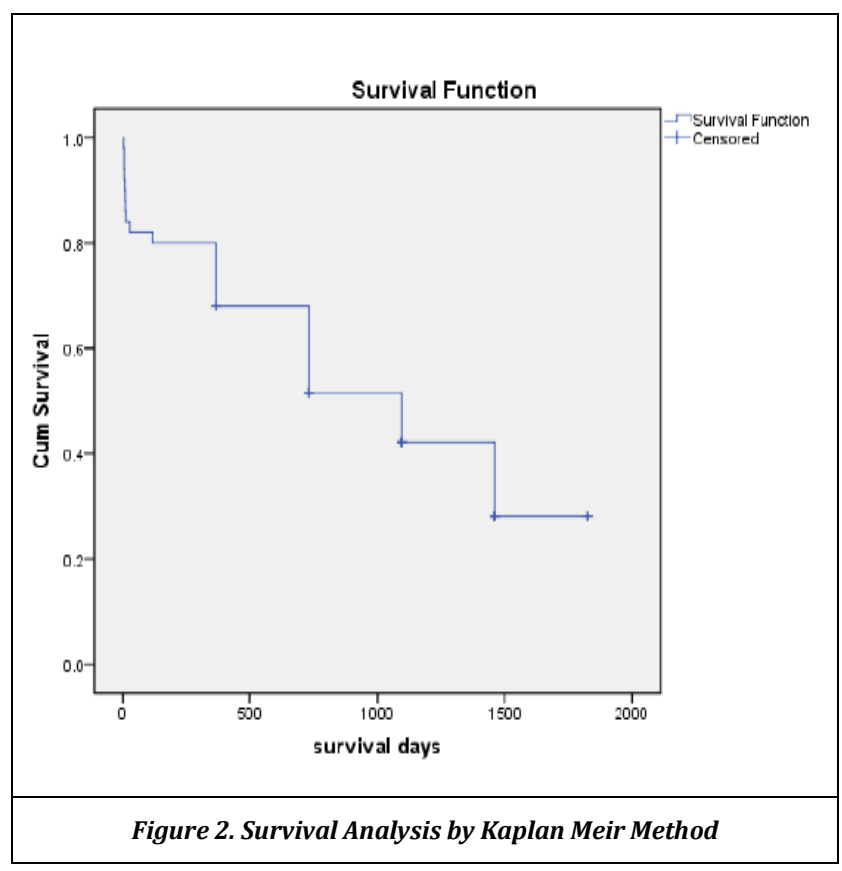

\section{DISCUSSION}

Regardless of the cell type, oesophageal cancer asserts aggressive biologic behaviour. With only two layers to the oesophageal wall, tumours rapidly infiltrate through the muscular wall into surrounding structures. The rich vascular and lymphatic supply facilitates spread to regional lymph nodes. Advanced disease is common at the time of 
presentation and contributes to the high mortality rate.

Various imaging techniques (Chest X-ray, barium swallow, computed tomography (CT), and positron emission tomography (PET) can determine the presence and extent of disease, however, the diagnosis of oesophageal cancer is based upon oesophageal biopsy.

Most of the study subjects fall in the age group $>60$ years and mean age is 59.3 years. In study done by Ferlay J et al on 116 patients with Carcinoma oesophagus in Europe, median age of 60 years (Range 36-81 years). ${ }^{8}$ Study group comprised of $36(72 \%)$ males and $14(28 \%)$ females, making M: F ratio of 1.89:1. This ratio compared favourably to the data for India based on the International agency for research on cancer (1.98:1) Smoking was found to be prominent risk factor in agreement with study by Ines Gockel et al. ${ }^{9}$ Smoking was found to have adverse effects on postop outcome due to increased chance of respiratory complications. As majority of the patients were well past their middle age, many of them had co-morbidities like HTN, DM and COPD. Diabetes had direct correlation to length of hospital stay among the high risk individuals though not significant enough. Majority of study group had Diabetes and hypertension. Incidence of COPD is $22 \%$ which was one of the causes of postoperative pulmonary complications.

$98 \%$ of the patients presented with dysphagia which is the most common symptom in oesophageal malignancy. The mean duration of onset of dysphagia to the surgery was 4.6 months which is earlier than 5-6 months reported by studies in Asia-Pacific region. High levels of literacy and good access to public health care in Kerala which is on par with most developed nations may be the reason

In the study most of the oesophageal malignancies were SCC type as noted by Baquet CR on Oesophageal cancer epidemiology. In our study majority of lesions in mid oesophagus were squamous cell carcinoma and presented in stage 3. In lower oesophagus $42.5 \%$ were in stage 2, where most were adenocarcinoma. Mortality rate was significantly higher in SCC according to study done by Ines Gockel et al. ${ }^{9}$ on Morbidity and mortality after oesophagectomy. But in our study, the mean survival days of both types of malignancy were comparable and not statistically significant.

Oesophageal malignancy has a rapid spread and often diagnosed late and in this study majority (52\%) of the cases presented at Stage 3. The mean survival days for stage 1, 2 \& 3 was obtained as 1147 days (37.7 months), 859 (28.24 months) days and 667 days (21.9 months) respectively.

It is difficult to get accurate information about outcomes for patients in different stages of oesophageal cancer, as most published clinical studies have pooled together the results of patients with stage I-III cancer. Most of the studies to date is based on 5-year survival rate. ${ }^{10}$

In one study from Japan, the average survival of 14 patients with stage II oesophageal cancer following surgery alone was 25 months which is comparable to our study

A small clinical study done by Texas Oncology group in 113 patients with stage III oesophageal malignancy, the average survival was 16 months for those who received combined treatment (Chemotherapy and surgery) and 11 months for those with surgery alone. ${ }^{11}$

Mean survival days of patients with oesophageal malignancy in our study is 799.68. Oesophageal malignancy diagnosed and treated at Stage 1 has survival advantage of
1.6 years compared to Stage 3. Stage 2 oesophageal malignancy has survival difference of 1.3 yrs., more than stage 3 and were statistically significant

However Stage 1 and 2 has only difference in survival of 116 days which is comparable. The results of a trial done in Texas indicate that in general, patients with stage II cancer experience a better than $20 \%$ survival and patients with stage III cancer experience a slightly worse outcome. According to American Cancer Society studies Stage at presentation has definite advantage over survival of patients which is comparable to our study. ${ }^{12}$

A large study done by Portale G et al in USA on resectable oesophageal carcinoma the overall five-year survival by stage was $81 \%$ for stage I, $51 \%$ for stage II, $14 \%$ for stage III, and $9 \%$ for stage IV. ${ }^{13}$

In our study pulmonary complications are the most important cause of postoperative morbidity (18\%) which is comparable to the studies by Mark. K. Furquson, Martin et al. ${ }^{14}$ Careful pre-operative assessment of pulmonary function and risk stratification go a long way in avoiding respiratory complications.

The mean survival of patients with complications was significantly reduced compared to those without complications. Those patients with any immediate complications had a reduced survival compared to those with no immediate postoperative complications.

Complications were present in $26.7 \%$ of cases who underwent Tri-incisional procedure and 35\% cases who underwent THE. In the study done by Ines Gockel et al. Univariate analysis identified the selection of the surgical procedure as the main risk factor affecting the mortality rate. ${ }^{9}$ Transthoracic oesophagectomy was associated with a higher immediate complication and morbidity than transhiatal dissection. ${ }^{15}$ In contrast to this, our study showed relatively less occurrence of complications in transthoracic procedure. No significant difference was demonstrated between these procedures, although the meaningfulness of these findings is limited due to the small number of patient enrolled and the lack of information on the oncological radicality, especially the extent of lymph node dissection. Among the 30 Transthoracic procedures 22 of them were Thoracoscopic, rest were converted to thoracotomy.

The fact that all transthoracic oesophagectomies require placement of chest tube for several days following surgery, coupled with problems faced with achieving full lung expansion, contributes to the prolonged stay ( $>13$ days) compared to the Transhiatal oesophagectomy. In our study the mean post-operative hospital stay was 10.69 for transhiatal compared to 17.33 for transthoracic group. In a study done by Hulscher et al in Netherlands on 50 patients TTE had hospital stay 21 days and they had 17 days hospital stay.

All the patients with preoperative serum albumin level $<3.5$ had anastomotic leak and was statistically significant. Quality of life is related to the nutritional support for surgery and essential for adequate immune function and wound healing. Studies by Ines Gockel et al., A M Ryan et al conclusively predict poor outcome for patients with hypoalbuminaemia9.

In a study done by Abbas Tabatabai on 61 patients treated for carcinoma of the oesophagus between 2006 and 2007 et al on incidence and risk factors predisposing to 
anastomotic leak $62.3 \%$ of their study population with anastomotic leak had serum albumin $<3.5 \mathrm{gm} / \mathrm{dl}$.

The average survival of patients treated with chemotherapy with surgery was 34 months, compared to 22 months for patients treated with surgery alone. Xiao Lyu and colleagues studied the survival benefits of adjuvant chemotherapy in patients underwent oesophagectomy. Postoperative taxane-based adjuvant chemotherapy improved survival of patients with lymph node positive thoracic ESCC compared with surgery alone.

On a follow up study done in 192 patients who underwent oesophagectomy by Manochechehr et al $100 \%$ of the study subjects had dysphagia. Among them $15.5 \%$ had Grade 1, $53.5 \%$ had grade 2 and 20.5 had grade 3 dysphagia ${ }^{16}$. In our study, $100 \%$ had dysphagia after surgery. Among them $57 \%$ had grade 3 dysphagia. This can be attributed the tumour recurrence problems related to gastrointestinal functional disorders after oesophageal resection and gastric tube reconstruction do not significantly impair long-term quality of life, which is mainly influenced by tumour recurrence.

\section{CONCLUSIONS}

Despite the magnitude of the operation and the comorbidities inherent to the cohort of patients presenting with oesophageal cancer, oesophageal resection can be accomplished successfully in selected patients with low mortality and acceptable morbidity. Considerable controversy continues regarding the best operation for a given patient, though a procedure that the surgeon can perform safely is certainly best. Since stage at presentation is a significant factor determining the survival of patients with oesophageal malignancy, early detection and diagnosis can significantly improve survival rate. Chemotherapy with surgery is considered a better option particularly in Stage 3 disease. Good nutritional status of patients is important to reduce the incidence of anastomotic leak.

\section{REFERENCES}

[1] Herszényi L, Tulassay Z. Epidemiology of gastrointestinal and liver tumors. Eur Rev Med Pharmacol Sci 2010;14(4):249-58.

[2] Lambert R, Hainaut P. The multidisciplinary management of gastrointestinal cancer. Epidemiology of oesophagogastric cancer. Best Pract Res Clin Gastroenterol 2007;21(6):921-45.
[3] Surveillance, epidemiology, and end results program turning. SEER Stat Fact Sheets: oesophageal Cancer. Retrieved November 9, 2013.

[4] Pennathur A, Gibson MK, Jobe BA, et al. Oesophageal carcinoma. Lancet 2013;381(9864):400-12.

[5] Absi A, Adelstein DJ, Rice T. oesophageal Cancer. 2013: p.

http://www.clevelandclinicmeded.com/medicalpubs/di seasemanagement/hematology-oncology/esophagealcancer/

[6] Siewert JR, Stein HJ, Feith M, et al. Histological tumor type is an independent prognostic parameter in oesophageal cancer: lessons from more than 1,000 consecutive resections at a single center in the Western world. Ann Surg 2001;234(3):360-9.

[7] Lerut T, Coosemans W, Decker G, et al. Surgical techniques. J Surg Oncol 2005;92(3):218-29.

[8] Ferlay J, Autier P, Boniol M, et al. Estimates of cancer incidence and mortality in Europe in 2006. Ann Oncol 2007;18(3):581-92.

[9] Gockel I, Exner C, Janginger T. Morbidity and mortality after oesophagectomy for oesophageal carcinoma: a risk analysis. World J Surg Oncol 2005;21:3:37.

[10] Lagergren J. Adenocarcinoma of oesophagus: what exactly is the size of the problem and who is at risk? Gut 2005;54(Suppl 1):i1-i5.

[11] Types of cancer - oesophageal cancer. Texas: Oncology society. 2017 June, 88.

[12] Early detection, diagnosis and staging of oesophageal cancer - survival rates of oesophageal cancer by stage. American Cancer Society, 2017 June 14:97.

[13] Portale G, Hagen JA, Peters JH, et al. Modern 5-year survival of resectable oesophageal adenocarcinoma: single institution experience with 263 patients. J Am Coll Surg 2006;202(4):588-98.

[14] Ferguson MK, Martin TR, Reeder LB, et al. Mortality after oesophagectomy. World J Surg 1997;21(6):599-604.

[15] Ellis FH Jr, Heatley GJ, Krasna MJ, et al. oesophagogastrectomy for carcinoma of the oesophagus and cardia: a comparison of findings and results after standard resection in three consecutive eight-year intervals with improved staging criteria. J Thorac Cardiovasc Surg 1997;113(5):836-48.

[16] Aghajanzadeh M, Safarpour F, Koohsari MR, et al. Functional outcome of gastrointestinal tract and quality of life after oesophageal reconstruction of oesophagus cancer. Saudi J Gastroenterol 2009;15(1):24-8. 